\title{
Diffractive orbits in quantum billiards
}

\author{
Nicolas Pavloff and Charles Schmit \\ Division de Physique Théorique円, Institut de Physique Nucléaire, F-91406 Orsay Cedex, France
}

\section{abstract}

We study diffractive effects in two dimensional polygonal billiards. We derive an analytical trace formula accounting for the role of non-classical diffractive orbits in the quantum spectrum. As an illustration the method is applied to a triangular billiard.

PACS numbers :

03.65.Sq Semiclassical theories and applications.

IPNO/TH 95-14 to to appear in Physical Review Letters

\footnotetext{
${ }^{1}$ Unité de Recherche des Universités Paris XI et Paris VI associée au CNRS.
} 
During the last decade several methods based on periodic orbit (PO) theory have been successfully employed to study quantum systems whose classical equivalent is chaotic (see e.g. [1]). PO theory applies also when the system is not fully hyperbolic (when some orbits appear in families [2]) or integrable [3]. More recently it has been refined to include complex orbits [4] and diffractive effects $[5,6$, 6]. In this line we aim at studying the problem of wedge diffraction as an extension of the standard PO theory. This is one of the oldest and simplest example of diffraction (see e.g. [7]) and it is also the case where the diffractive corrections to semiclassics are the more important.

In this Letter we calculate for the first time the role of non-classical diffractive orbits in the spectrum of two-dimensional polygonal billiards. We derive a trace formula embodying the contribution of diffractive PO's to the level density (Eq. (9)). This contribution is of order $\sqrt{\hbar}$ smaller than the contribution of isolated PO's and is the next order term in the trace formula. As an example the formalism is applied to a triangular billiard with angles $(\pi / 4, \pi / 6,7 \pi / 12)$ and one sees that it provides a very accurate description of the Fourier transform of the spectrum.

We consider a quantum particle enclosed in a polygonal billiard $\mathcal{B}$ and we impose Dirichlet boundary conditions on the frontier $\partial \mathcal{B}$. Hence the associated Green function is solution of the following equation :

$$
\begin{aligned}
\left(\Delta_{B}+k^{2}\right) G\left(\mathbf{q}_{B}, \mathbf{q}_{A}, k\right) & =\delta\left(\mathbf{q}_{B}-\mathbf{q}_{A}\right) \quad \text { inside } \mathcal{B}, \\
G\left(\mathbf{q}_{B}, \mathbf{q}_{A}, k\right) & =0 \quad \text { on } \partial \mathcal{B} .
\end{aligned}
$$

where $\mathbf{q}$ is a coordinate in configuration space.

The semiclassical approximation for $G$ reads (see e.g. [8])

$$
G_{0}\left(\mathbf{q}_{B}, \mathbf{q}_{A}, k\right)=\sum_{\mathbf{q}_{A} \rightarrow \mathbf{q}_{B}} \frac{\mathrm{e}^{i(k L-\mu \pi / 2)}}{i \sqrt{8 i \pi k L}},
$$

where the sum is taken over all classical trajectories going from $\mathbf{q}_{A}$ to $\mathbf{q}_{B}$. In (2) $L$ is the length of the trajectory and $\mu$ is the associated Maslov index [8]. In polygonal enclosures the boundary has no focussing components, there are no caustics and $\mu$ is simply twice the number of bounces of the trajectory on $\partial \mathcal{B}$.

In polygonal billiards the hamiltonian flow is discontinuous on the vertices [9] and when the angle at a vertex is not of the form $\pi / n\left(n \in \mathbb{N}^{*}\right)$ this causes diffraction (see e.g. [10]). Then, following Keller's geometrical theory of diffraction [11], one is lead to consider non-classical contributions to the Green function which are "diffractive orbits" starting at $\mathbf{q}_{A}$, going to a vertex $\mathbf{q}_{1}$ and then to $\mathbf{q}_{B}$. These orbits are non classical because at $\mathbf{q}_{1}$ the reflection is not specular. Far from the region of discontinuity of the hamiltonian flow the corresponding Green function may be taken to be :

$$
G_{1}\left(\mathbf{q}_{B}, \mathbf{q}_{A}, k\right)=G_{0}\left(\mathbf{q}_{1}, \mathbf{q}_{A}, k\right) \mathcal{D}_{1}\left(\theta, \theta^{\prime}\right) G_{0}\left(\mathbf{q}_{B}, \mathbf{q}_{1}, k\right),
$$

where $\mathcal{D}_{1}\left(\theta, \theta^{\prime}\right)$ is a diffraction coefficient evaluated in the solvable case of two semi infinite straight lines meeting with an angle $\gamma$ equal to the interior angle of the polygon at $\mathbf{q}_{1} \cdot \theta$ (resp. $\theta^{\prime}$ ) is the angle of the incoming (resp. outcoming) trajectory at $q_{1}$ with the boundary. $\mathcal{D}_{1}\left(\theta, \theta^{\prime}\right)$ reads [10, 11, 12] : 


$$
\mathcal{D}_{1}\left(\theta, \theta^{\prime}\right)=-\frac{4}{N} \frac{\sin (\pi / N) \sin (\theta / N) \sin \left(\theta^{\prime} / N\right)}{\left(\cos \frac{\pi}{N}-\cos \frac{\theta+\theta^{\prime}}{N}\right)\left(\cos \frac{\pi}{N}-\cos \frac{\theta-\theta^{\prime}}{N}\right)},
$$

where $N=\gamma / \pi$ is not assumed to be an integer.

As stated above one sees in expression (目) that when $\gamma$ is of the form $\pi / n, \mathcal{D}_{1}$ is zero and there is no diffraction. Indeed in this case a trajectory passing by $\mathbf{q}_{1}$ is the limit of a trajectory bouncing specularly $n$ times near the vertex and a contribution of type (2) accounts for the effect of the wedge. This is to be related to the fact that in this case there exists a $n^{t h}$ iterate of the flow which is continuous [9]. Note also that $\mathcal{D}_{1}$ is zero if $\theta$ or $\theta^{\prime}$ is equal to 0 or $\gamma$ (i.e. in the case of a diffractive trajectory having a segment lying on a face).

For an orbit with several diffractive reflections at points $\mathbf{q}_{1} \ldots \mathbf{q}_{\nu}$ formula (3) becomes

$$
G_{\nu}\left(\mathbf{q}_{B}, \mathbf{q}_{A}, k\right)=G_{0}\left(\mathbf{q}_{1}, \mathbf{q}_{A}, k\right)\left\{\prod_{j=1}^{\nu-1} \mathcal{D}_{j} G_{0}\left(\mathbf{q}_{j+1}, \mathbf{q}_{j}, k\right)\right\} \mathcal{D}_{\nu} G_{0}\left(\mathbf{q}_{B}, \mathbf{q}_{\nu}, k\right),
$$

where $\mathcal{D}_{j}$ is the diffraction coefficient at point $\mathbf{q}_{j}$ as given by (4).

In (2), (3) and (5) the indices 0,1 or $\nu$ of the Green function recall that diffractive effects are subdominant (by a factor of order $k^{-\nu / 2}$ ). There might be less severe non analyticities on the boundary leading to higher order diffractive corrections. Note also that we are using here a simple approximation for the Green function which is not valid when the angles $\theta$ and $\theta^{\prime}$ at an edge are such that the diffractive orbit is close to be real ; in this case the coefficient $\mathcal{D}_{1}\left(\theta, \theta^{\prime}\right)$ diverges. This occurs in vicinity of the line of discontinuity of the hamiltonian flow. In order to have a formula valid in all regions of space one should use a uniform approximation such as first provided by Pauli [12 and whose general form is given in [10] (see also [13]).

The level density $\rho(k)$ is then obtained from the Green function by the usual formula :

$$
\rho(k)=-\frac{2 k}{\pi} \operatorname{Im} \int_{\mathcal{B}} d^{2} q G(\mathbf{q}, \mathbf{q}, k) .
$$

$\rho(k)$ can be separated in a smooth function of $k, \bar{\rho}(k)$ plus an oscillating part $\widetilde{\rho}(k)$. The zero length trajectories in (6) contribute to $\bar{\rho}$ and will not be considered in detail here (see [14]). When $G$ is replaced by its semiclassical approximation (2) a stationary phase evaluation of (6) corresponds in considering only the contribution of classical PO's to $\widetilde{\rho}$. When diffractive orbits such as (5) are taken into account one is lead to consider also "diffractive PO's" which are PO's with one or several diffractive reflections (example of such orbits are given on Fig. 1).

Let us consider first the contribution of classical PO's. In a polygonal enclosure there is a drastic difference between PO's with even and odd number of bounces. The latter ones do not remain periodic when a point of reflection is translated along a face (they period-double into a PO with twice as many bounces). This can be understood by remembering that, for the phase-space coordinates transverse to the direction of an orbit, a bounce on a straight segment leads to an inversion. On the other hand, PO's with an even number of bounces form families which correspond to local translation parallel to the faces of the polygon. They are neutral (or direct parabolic, see [8]) PO's to which the usual trace formula does not apply ; we use a generalization of Gutzwiller theory which is valid for the case of 
degenerate PO's [2]. We quote here the result and leave detailed discussion for the future [13]. A family of orbits contributes to $\widetilde{\rho}(k)$ as :

$$
\widetilde{\rho}(k) \leftarrow \sqrt{\frac{k L}{2 r \pi^{3}}} d_{\perp} \cos (k r L-\pi / 4) .
$$

(7) is written for the general case of the $r^{t h}$ iterate of a primitive orbit of length $L\left(r \in \mathbb{N}^{*}\right)$. $d_{\perp}$ is the length occupied by the family perpendicular to the orbit's direction. It is equal to $d \cos \phi$, where $d$ is the length occupied by the family on a face and $\phi$ the angle between the direction of the orbit and the normal to this face.

For an isolated PO with an odd number of bounces one has the following contribution :

$$
\widetilde{\rho}(k) \leftarrow-\frac{L}{2 \pi} \cos (k r L) .
$$

Formula (8) holds when the number of repetitions is odd. When $r$ is even, the $r^{\text {th }}$ iterate of an isolated orbit leads to a family and formula (7) applies.

The derivation of the contribution of a diffractive PO is patterned on what is done in Gutzwiller's trace formula for an isolated PO. The length of a closed diffractive orbit in the vicinity of the diffractive PO is expanded up to second order and the trace of the Green function is evaluated by a stationary phase approximation. The final contribution of a generic diffractive PO with $\nu$ diffractive reflections to the oscillating part of the level density reads :

$$
\widetilde{\rho}(k) \leftarrow \frac{L}{\pi}\left\{\prod_{j=1}^{\nu} \frac{\mathcal{D}_{j}}{\sqrt{8 \pi k L_{j}}}\right\} \cos (k L-\mu \pi / 2-3 \nu \pi / 4) .
$$

In (9) $L_{1} \ldots L_{\nu}$ are the lengths along the orbit between two diffractive reflections. $L_{1}+\ldots+L_{\nu}=L$ is the total length of the diffractive PO. $\mu$ is the Maslov index which is here twice the number of specular reflections. Formula (9) is the most important result of this paper. Note that different diffractive orbits may combine if they have diffraction points in common. Hence repetitions of a primitive diffractive orbit appear as a special case of (9) ; in this case however, in the first factor $L / \pi$ of the r.h.s. of (9), $L$ should be understood as the primitive length of the orbit. The above formulae show that the contribution of a family of orbits in of order $\mathcal{O}\left(k^{1 / 2}\right)$, for an isolated orbit it is $\mathcal{O}(1)$ and for a diffractive PO it is $\mathcal{O}\left(k^{-\nu / 2}\right)$. Nevertheless we will see in the following that diffractive orbits have a very noticeable contribution to the level density.

We will now illustrate our approach by studying a specific example. Let us consider a triangle with angles $(\pi / 4, \pi / 6,7 \pi / 12)$. As explained above diffraction occurs only at the vertex with angle $7 \pi / 12$. The scale of lengths and wave-vectors is fixed by the value $h$ of the height going from this vertex to the opposite face. We take $h=1$ in the following. The shortest classical and diffractive PO's in this triangle are shown on Fig. 1. Diffractive reflections are indicated with a black spot. Note that the first orbits are diffractive, classical orbits (isolated or in families) occur at greater lengths. The spectrum was computed numerically by expanding the wave function around the vertices with angles $\pi / 4$ and $\pi / 6$ in "partial waves" which are Bessel functions with a sinusoidal dependance on the angle defined near the vertex considered. More precisely if $r_{n}$ and $\varphi_{n}$ are polar coordinates defined near the vertex $\pi / n(n=4$ or 6$)$ the partial waves in this region are of the form $J_{n m}\left(k r_{n}\right) \sin \left(n m \varphi_{n}\right)$ with $m \in \mathbb{N}^{*}$. 
One then imposes matching of the wave function and of its first derivative along the height $h$ (see details in [13]). We determined the first 957 levels, up to $k_{\max } \simeq 96$. The accuracy of the computation was tested by varying the number of matching points and of partial waves. We evaluate the typical error on an eigenvalue as being of order of a hundredth of the mean level spacing.

In order to visualize the importance of classical and diffractive PO's of successive lengths in the spectrum we study the regularized Fourier transform of the level density :

$$
F(L)=\int_{0}^{k_{\max }} k \mathrm{e}^{i k L-\alpha k^{2}} \rho(k) d k .
$$

If $k_{\max } \rightarrow+\infty$ and if the regularizing coefficient $\alpha$ is set to zero in (10) $F(L)$ is just a series of delta peaks centered on the lengths of the classical and diffractive PO's. The multiplicative factor $k$ in (10) is meant to cancel the singularity $k^{-\nu / 2}$ of the contribution of a diffractive PO of type (9) with up to $\nu=2$ diffractive reflections. We take here $\alpha=9 / k_{\text {max }}^{2}$ and plot $|F(L)|$ on Fig. 2. The numerical result is represented by a thin line and the semiclassical approach (7,8) corrected by diffractive PO's (9) by a thick line. We also included the contribution of $\bar{\rho}(k)$ in order to reproduce the initial peak at $L=0$. We see that the agreement is excellent. Note that the existence of diffractive PO's is of great importance for reproducing all the peaks in $|F(L)|$. This is illustrated on the figure where their contribution (9) has been dashed.

Here several comments are in order. Note first that the diffractive PO's labelled 2 and 4 on Fig. 1 have not been included because their diffraction coefficient is zero. Also the orbit labelled 7 on Fig. 1 has a non standard contribution : it is an isolated orbit which accounts for boundary effects on the family with the same length (labelled 6 on Fig. 1). In addition to the orbits of this family it has an extra reflection on the bottom face (the same type of orbit was considered in Ref. [15, 16]). The weight of PO number 7 is reduced by a factor $1 / 2$ compared to (8) since one integrates only over closed orbits on one side of this limiting PO. Also we included repetitions of diffractive PO's number 1 and 3 and they can be seen to have still a noticeable contribution. We did not include the diffractive PO composed by the sum of orbit 1 and 3 although it can be considered as a small diffractive correction to the contribution of family 6 . Indeed the orbit " $1+3$ " lies just on the region separating real orbits from diffractive ones and as mentioned above it can not be accounted for by a simple diffraction coefficient such as (4). This type of corrections will be treated in a forthcoming publication [13].

To summarize let us emphasize the important role of non classical orbits in the spectrum of quantum billiards. The existence of these orbits affects qualitatively the Fourier transform of the spectrum. The above example is only one among others were the discontinuity of the classical dynamics is linked to strong diffractive corrections to semiclassics. It was argued in [17] that the same type of corrections should be taken into account for the 3 dimensional icosahedral billiard. We expect also diffractive effects - of the same order as those described here - in more general billiards with cusps (non-polygonal or with an additional external field) ; in these cases a simple generalization of formula (9) accounts for the role of diffractive PO's. We note finally that the present work illustrates that semiclassical methods provide a very appealing tool which, when corrected with tunneling or diffractive effects, allows to describe accurately the solution of partial differential equations using simple geometrical methods.

It is a pleasure to thank E. Bogomolny and D. Ullmo for fruitful discussions. Division de Physique Théorique is a Unité de Recherche des Universités de Paris XI et Paris VI associée au CNRS. 


\section{References}

[1] for a review see Chaos and Quantum Mechanics, edited by M. J. Giannoni, A. Voros and J. Zinn-Justin, les Houches summer school lectures LII, (North Holland, Amsterdam, 1989) and also Chaos 2 1-158 (1992).

[2] S. C. Creagh and R. G. Littlejohn, Phys. Rev. A 44, 836 (1991) ; J. Phys. A. 25, 1643 (1992).

[3] M. V. Berry and M. Tabor, Proc. R. Soc. Lond. A 349, 101 (1976) ; J. Phys. A 10, 371 (1977).

[4] J. M. Robbins, S. C. Creagh and R. G. Littlejohn, Phys. Rev. A 39, 2838 (1989) ; M. Kuś, F. Haake and D. Delande, Phys. Rev. Lett. 71, 2167 (1993) ; P. Lebœuf and A. Mouchet, Phys. Rev. Lett. 73, 1360 (1994).

[5] G. Vattay, A. Wirzba and P. E. Rosenqvist, Phys. Rev. Lett. 73, 2304 (1994).

[6] N. D. Whelan "Geometric and diffractive orbits in the scattering from cofocal hyperbolae", preprint december 1994.

[7] A. Sommerfeld, Optics (Academic Press, New-York, 1954).

[8] M. C. Gutzwiller, Chaos in Classical and Quantum Mechanics (Springer-Verlag, New-York, 1990).

[9] T. Dagaeff and C. Rouvinez, Physica D 67, 166 (1993).

[10] G. L. James Geometrical Theory of Diffraction for Electromagnetic Waves (Peregrinus, Stevenage, 1976).

[11] J. B. Keller, J. Opt. Soc. Amer. 52, 116 (1962).

[12] W. Pauli, Phys. Rev. 54, 924 (1938).

[13] N. Pavloff and C. Schmit, in preparation.

[14] H. Baltes and E. R. Hilf, Spectra of Finite Systems (Bibliographisches Institut, Mannheim, 1976).

[15] B. Lauritzen, Phys. Rev. A 43, 603 (1991).

[16] M. Sieber, U. Smilansky, S. C. Creagh and R. G. Littlejohn, J. Phys. A 26, 6217 (1993).

[17] N. Pavloff and S. C. Creagh, Phys. Rev. B 48, 18164 (1993). 


\section{Figure captions}

Figure 1. The shortest classical and diffractive PO's in the triangle $(\pi / 4, \pi / 6,7 \pi / 12)$. All these orbits are self-retracing. For diffractive PO's the diffraction point is marked with a black spot. Orbits 6 and 10 form families, 5 and 7 are isolated. The lengths are given in unit of the height of the triangle.

Figure 2. $|F(L)|$ as a function of $L$. The thin line is the numerical result and the thick line the semiclassical approximation (77,8) with diffractive corrections (91). The two curves are hardly distinguishable. The contribution of the diffractive PO's has been dashed. 